\title{
Analysis of twitter data for India and China Covid-19 Vaccination
}

\section{Ms. Chesta Dhingra ${ }^{1}$, Mr. Satyabrata Sahoo ${ }^{2}$, Prof. Y.K. Gupta ${ }^{3} \&$ Mr. Amogh Dev Rai ${ }^{4}$}

\section{Objective}

Novel Corona Virus Disease, also known as COVID-19, is declared as a global pandemic by the World Health Organization on 11th March 20205. Since then, this virus has infected 108,822,960 people globally ${ }^{6}$. Further, the pandemic had killed around 2.4 million people till 16th February 2021, as per the World Health Organization report ${ }^{7}$. Thus, to combat this pandemic there is an urgent requirement of vaccination. With deployment of vaccinations like Astrazeneca, Covaxin and Sinopharam from India and China respectively, we can somewhere overcome this battle against SARS novel coronavirus.

The main aim behind writing this paper is to know the consensus or the opinion of people on the global scale towards Indian and Chinese vaccination drive by doing a comparative analysis with the help of twitter data and global media reviews (which includes the textual data other than Indian and Chinese media).

\section{Methodology}

While doing the twitter and global media reviews analysis, we have applied the Natural Language Processing method. Sentiments have been classified into three segments: positive, negative and neutral. Besides, analysing the sentiments, evaluation of frequencies of the words has been done in the wordcloud format. Further analysis of the most frequent bigrams ${ }^{8}$ and trigrams ${ }^{9}$ for India and China COVID-19 vaccination has been done from the twitter data. In the study we have included certain keywords like "covid-19 vaccination India", "covid-19 vaccination China" and "covidvaccination" before collecting the textual data from twitter API.

After doing the data collection (here, retweets have not been considered), pre-processing or cleaning of textual data has been done which includes removal of all the unnecessary characters like punctuations, English common words or stopwords for example a, an, the, is, am, can etc., alpha-numeric characters,

\footnotetext{
${ }^{1}$ Principal Author, Deputy Manager, open Data Weather Lab, Sanrachana

${ }^{2}$ Corresponding author, Technical Lead, Sanrachana

${ }^{3}$ Secondary author, Advisor, THSTI, Department Biotechnology, Government of India

${ }^{4}$ Secondary author, Executive Director, Sanrachana, senior academic fellow, SGT University

${ }^{5} \mathrm{https}$ ///link.springer.com/article/10.1007/s10489-020-02029-z

${ }^{6} \mathrm{https}$ ://covid19. who.int/?gclid=CjwKCAiAmrOBBhA0EiwArn3mfCD2HbI5xE0GZY9QH3-M8EiNzR-

ccKDop43OOivSkWzvPsdetPKgrBoC6XIQAvD_BwE

${ }^{7}$ https://covid19. who.int/?gclid=CjwKCAiAmrOBBhA0EiwArn3mfCD2HbI5xE0GZY9QH3-M8EiNzRccKDop43OOivSkWzvPsdetPKgrBoC6XIQAvD_BwE

${ }^{8}$ Sentences or the text corpus tokenized into pair of words which is called as bigrams.

${ }^{9}$ The text corpus tokenized into three words which is known as trigrams.
} 
urls, mentions and hashtags. The text pre-processing is an essential step to find good and accurate results of the unstructured data.

Top words in n-grams $(\mathrm{n}=1,2,3)$ have been visualized, first on the global basis using the wordcloud, second, visualizing the bar plots for the top 50 bigrams and trigrams and last, doing the comparison analysis of sentiments.

The sentiments which are also referred as polarity and its range lies between $[-1,1]^{10}$ where 1 stand for the positive statement (where people have positive sentiments or feeling), 0 for the neutral statement (where people are bit sceptical, regarding the topic which they are referring to) and last -1 or below 0 are negative statements (where people are having negative opinions or statement regarding that topic).

To avoid the biasness towards the positive sentiments, we have categorised the polarity or sentiments into three segments. Here, personal opinions, judgements and emotions are referred to as Subjectivity of the statements for which the range lies between $(0,1)$. Whereas the factual information is referred to as objective/aim of the statements.

The tools that have been used while doing the research are R and Python with the help of certain packages like twitteR, ROAuth (both the packages are available in R) and python libraries wordcloud, pandas, NLTK, numpy, textblob, matplotlib, twint and tweepy.

\section{Observations}

First, according to the global observations for which we have extracted approximately 36000 tweets out of which we are able to analyse approx. 24000 tweets (rest of the twitter statements are not in the English language). Among these 24000 tweets, approximately $54.948 \%$ of the tweets are falling under the category of positive statement for which we can say that more than half of the tweets have positive notion towards the vaccination. $30.25 \%$ of people have the neutral opinion towards the covid-19 vaccination and only $14.79 \%$ of the twitter statements are having pessimistic approach towards the covid-19 vaccination exercise.

Besides the polarity, the factual or the objective information in the text data is around $24.90 \%$ and rest of the statements are subjective, which means most people are giving their judgments, opinions regarding the mass covid-19 vaccination drive.

Fig1 is showing the first 5000 most frequently occurring words in the Global data tweets. It shows that how the people at the global level are making the statements towards the vaccination. It also shows which are the most frequent words that people are using regarding coronavirus vaccination. Fig2 and

\footnotetext{
${ }^{10}$ https://buildmedia.readthedocs.org/media/pdf/textblob/latest/textblob.pdf
} 
Fig3 are the graphical representations of numbers and percentages of tweets that have been classified into positive, neutral and negative statements.

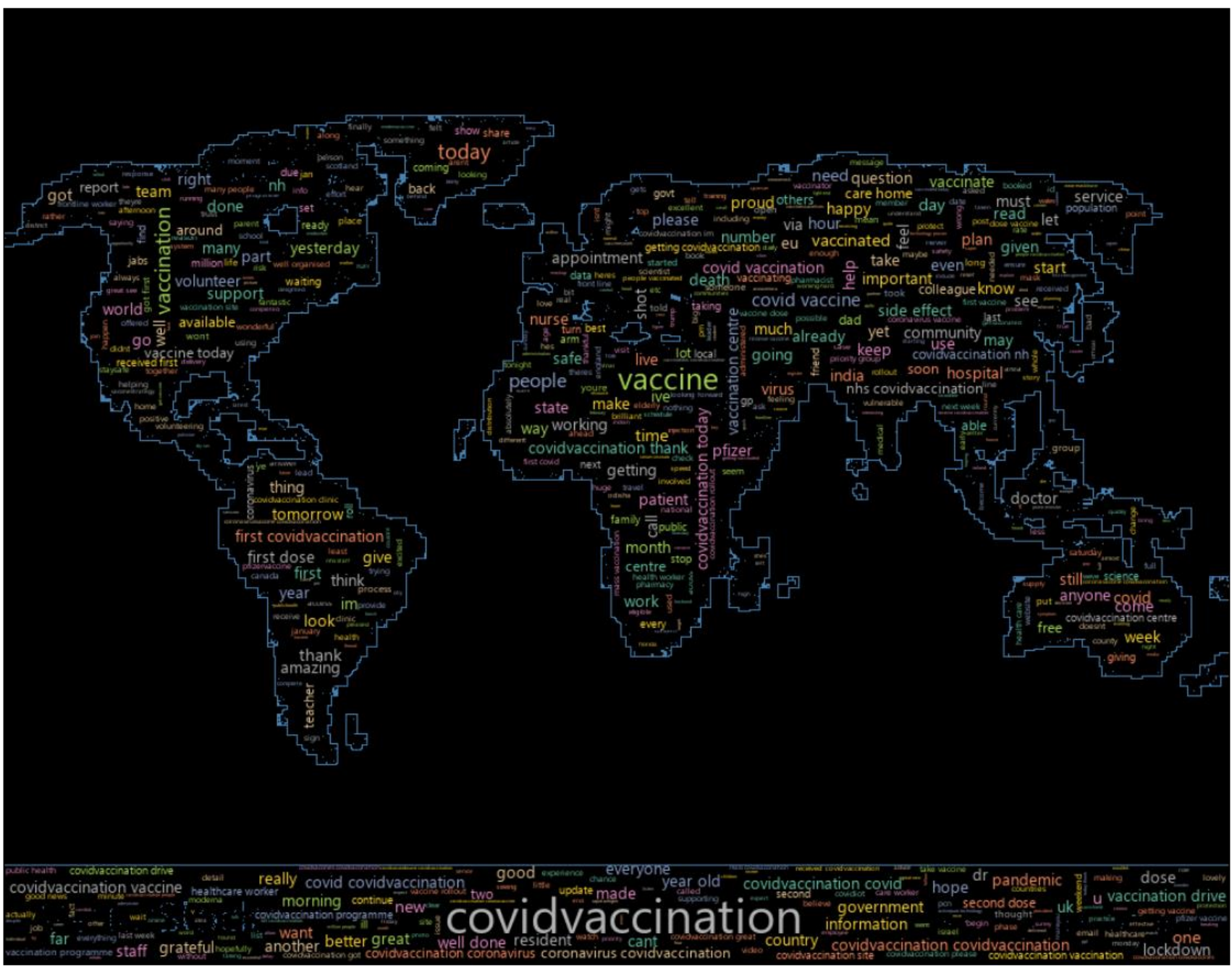

Figure 1 Word cloud of first 5000 the most frequent words from the Global Vaccine data

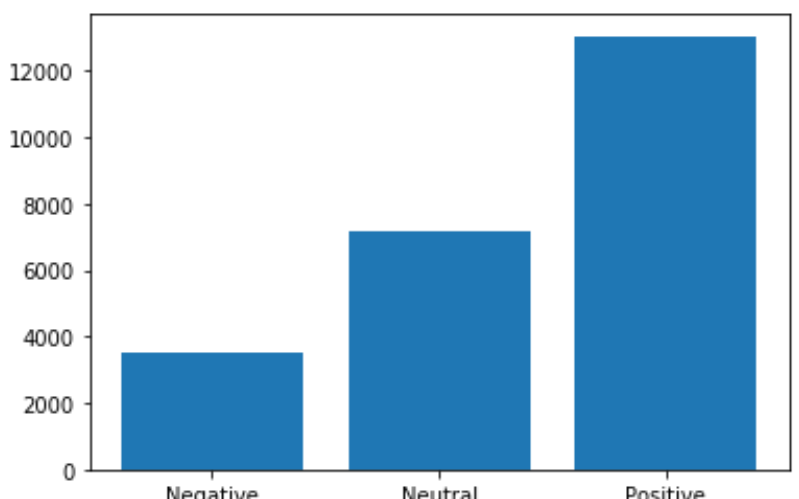

Figure 2 Graphical representation of number of global data tweets that have been classified into three segments positive, negative and neutral.

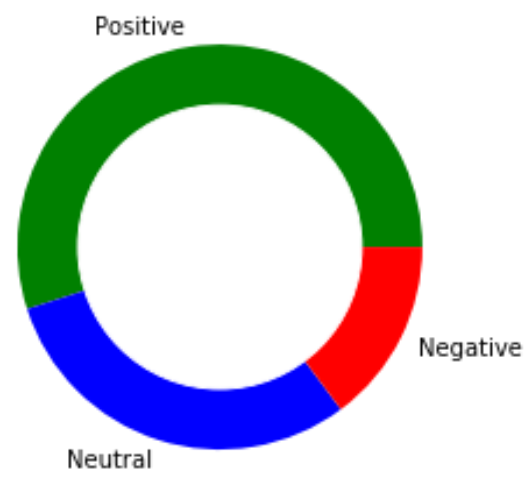

Figure 3 Graphical representation of percentage of tweets that have been categorised into positive, neutral and negative statements. 
The comparative analysis on the tweets for both the countries shows that for India out of 20,826 tweets $34.11 \%$ of people have positive sentiments about it whereas for China out of 7,568 tweets approximately $40.42 \%$ of positive tweets have been recorded. People have more of neutral opinions for Indian vaccination which is approximately $53.83 \%$ whereas for China it is $45.90 \%$ and lastly around $12.05 \%$ of the negative sentiments have been made for Indian vaccination whereas for China it is approximately 13.67\%. Fig 4 and Fig 5 is showing the word cloud of first 1500 words that occurred most frequently in both the India and China vaccination tweets dataset.

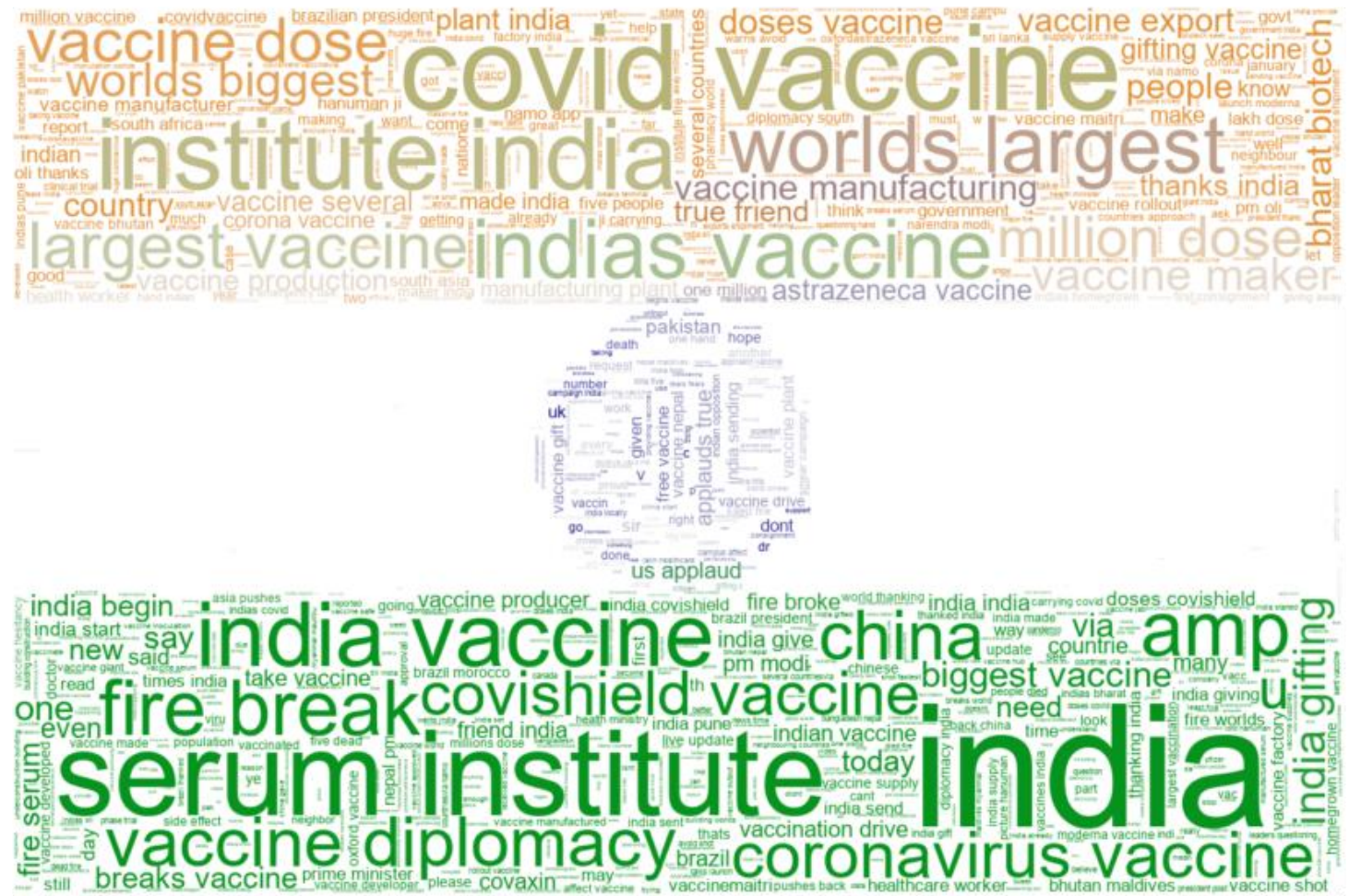

Figure 4 Word cloud of first 1500 the most frequent words. According to this the most talked vaccine is the one developed by Serum Institute of India 


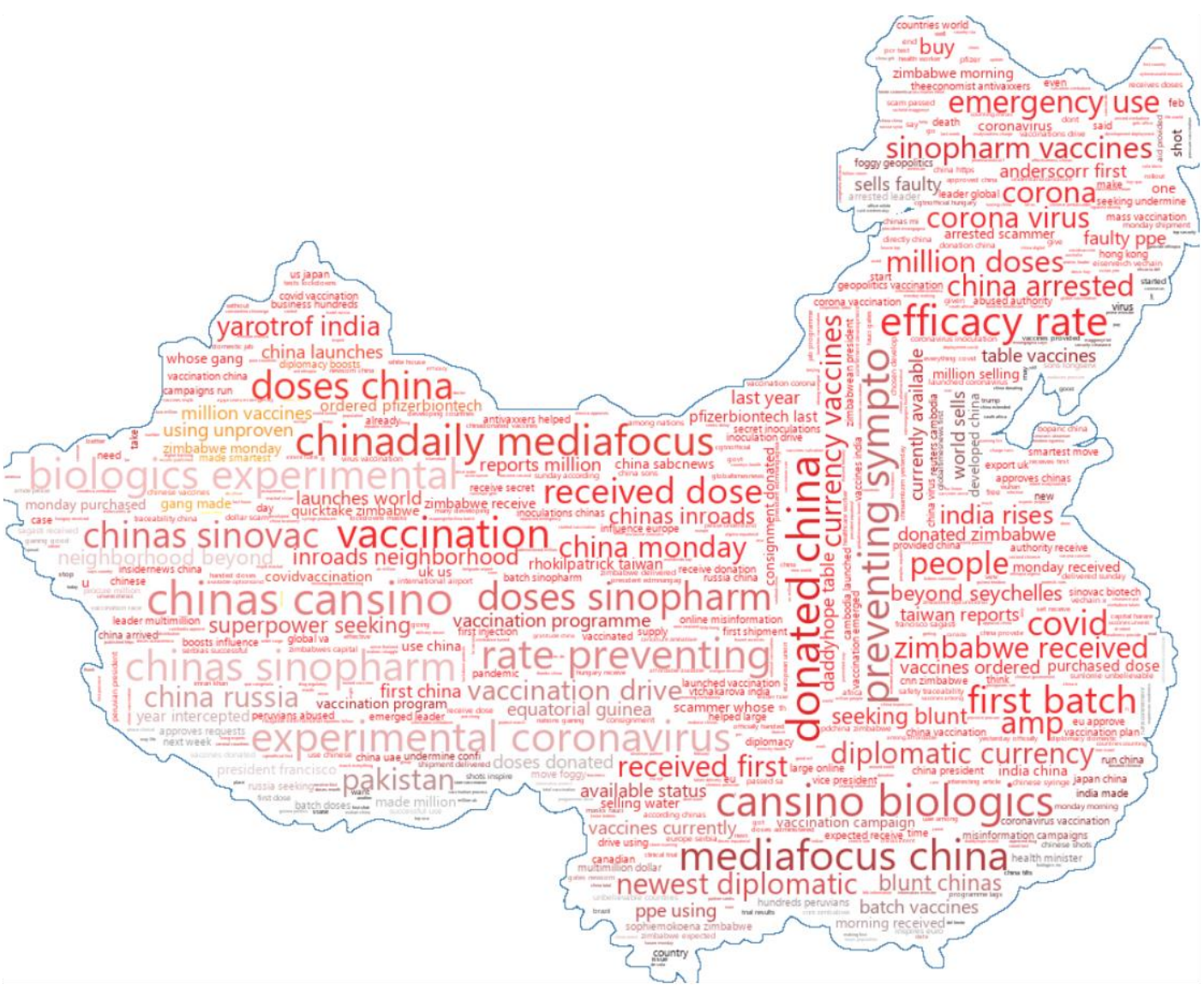

Figure 5 Word Cloud of China Vaccination data with maximum word frequencies of 1500 in which some of the common words other than chinas, vaccines and vaccination are doses, efficacy rate, Pakistan, sinopharm vaccines, faulty ppe, biologics, zimbawe.

Fig 6, Fig 7 and Fig 8, Fig 9 are the bar plots \& pie chart of the tweets that have been classified as positive, neutral and negative for both the countries India and China.

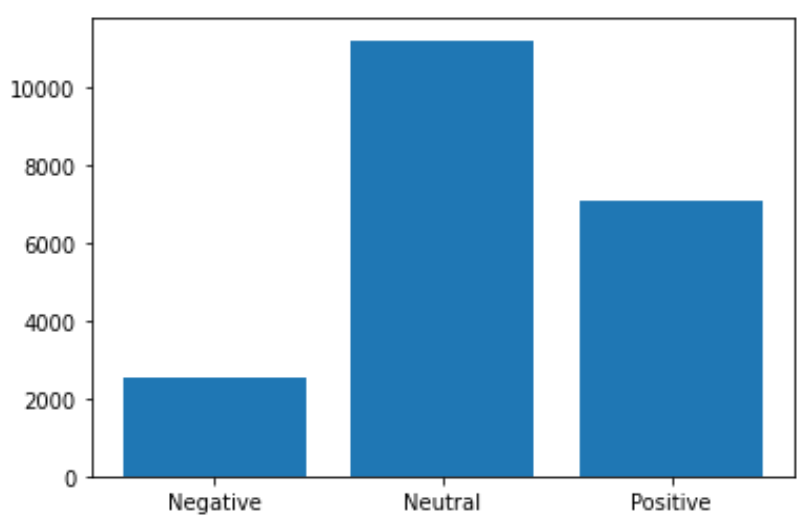

Figure 6 Bar plot of Sentiment Analysis of tweets for India Covid-19 Vaccination

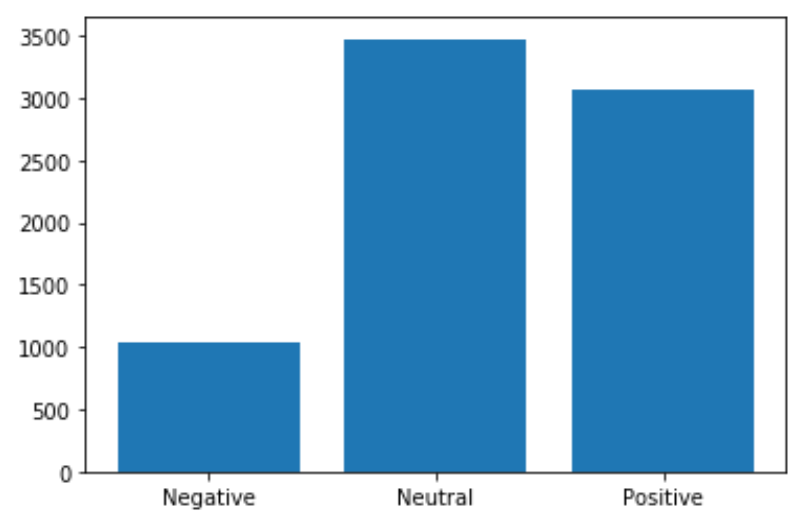

Figure 7 Bar plot of Sentiment Analysis of tweets for China Covid-19 vaccination 


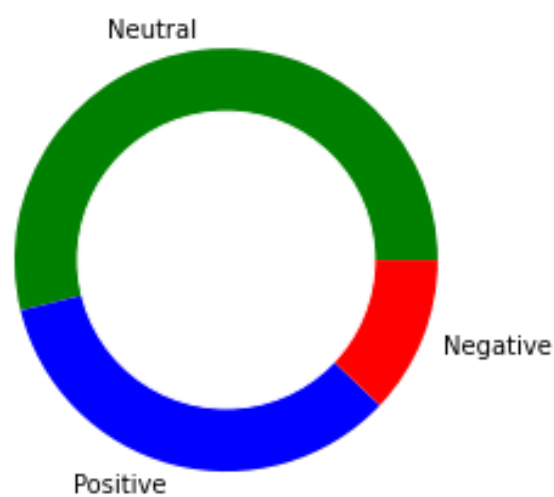

Figure 8 Percentage of polarity for Indian Vaccination tweets pie chart

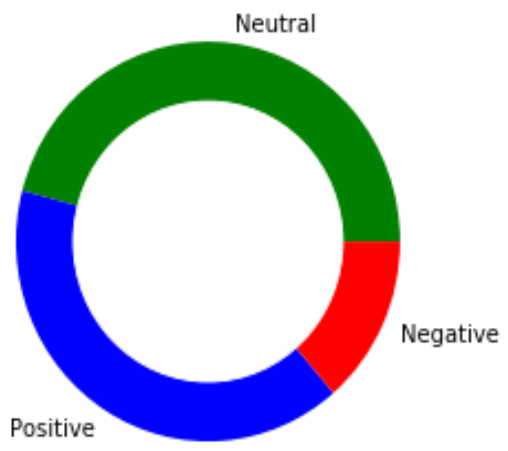

Figure 9 Percentage of polarity for China Vaccination tweets pie chart.

Above graphs show the polarity of the complete sentences present in the dataset for both the countries. Besides the polarity, only $47.58 \%$ of the tweets contain the factual information for India and rest are the opinions or judgements of people regarding India coronavirus vaccination whereas in China $40.53 \%$ are the facts or the information presented in the dataset and rest are the opinions and judgments presented by the people all around the world.

Further, breaking down the analysis (Fig 10, Fig 11, Fig 12, Fig 13, Fig 14, Fig 15, Fig 16, and Fig 17) in terms of bigram and trigrams for both the countries we can conclude that most of the statements are in neutral formats.

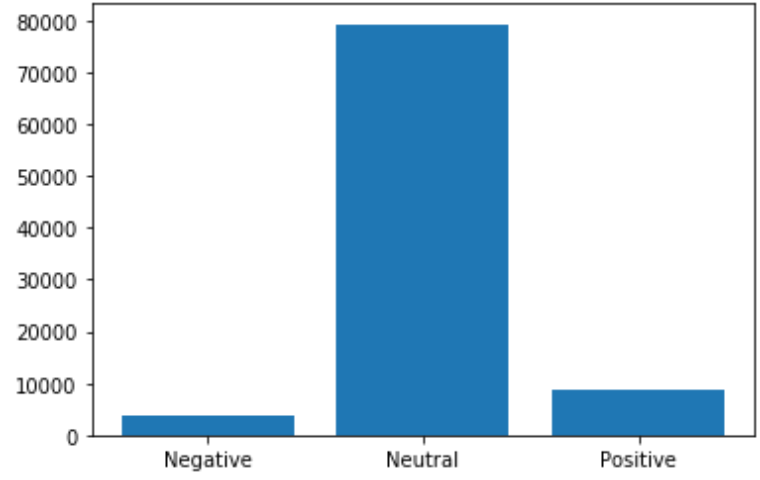

Figure 10 Numerical representation of polarity of the bigrams for Indian Vaccination

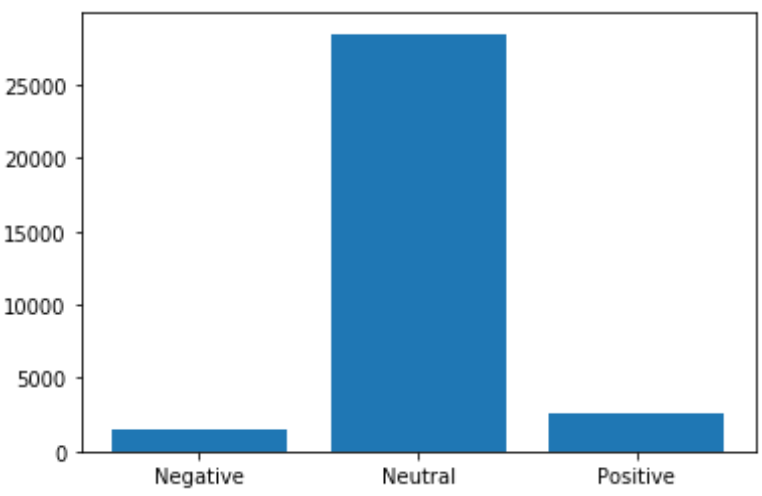

Figure 11 Numerical representation of polarity of the bigrams for China Vaccination 


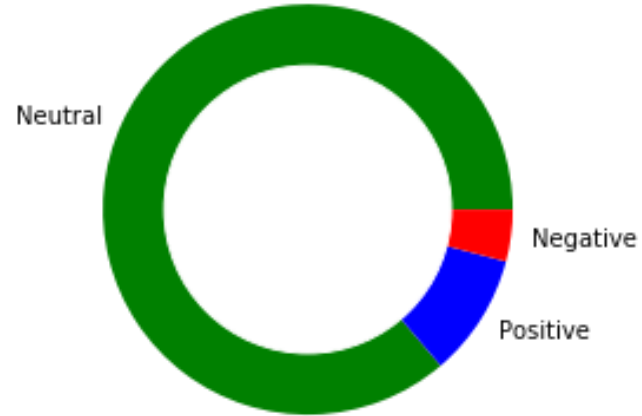

Figure 12 Polarity percentage of bigram for India Covid-19 vaccination. In this approximately $86.23 \%$ are the neutral statements out of 91,808 corpora, $9.69 \%$ are the positive and $4.08 \%$ are the negative statements.

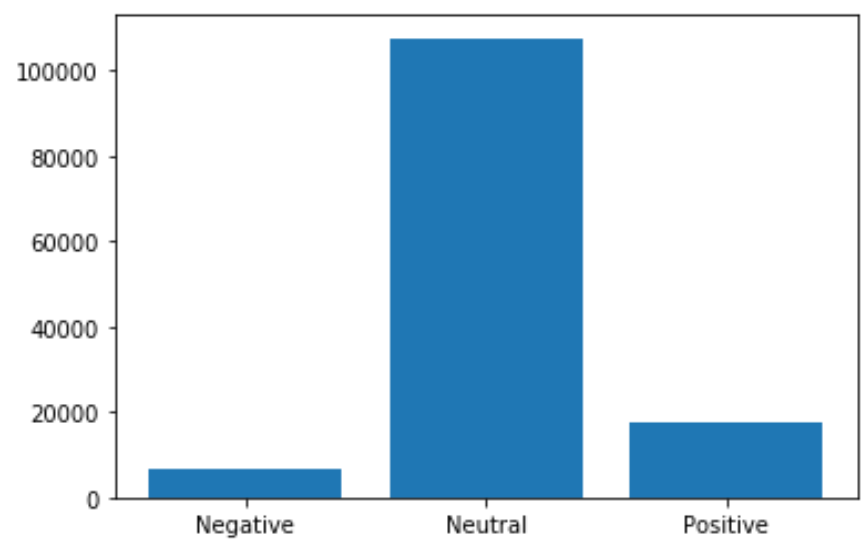

Figure 14 Graphical representation of number of trigrams that have been classified into three different segments negative, positive and neutral in the Indian Covid-19 vaccination context

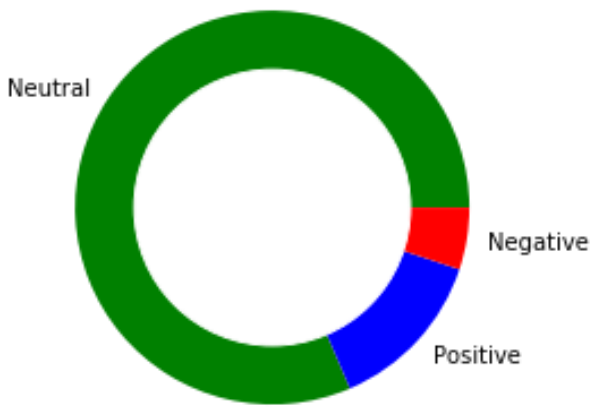

Figure 16 Polarity percentage of trigram for India Covid-19 vaccination. In this approximately $81.46 \%$ are the neutral statements out of approximately 1 lakh corpora, $9.69 \%$ are the positive and $4.08 \%$ are the negative statements.

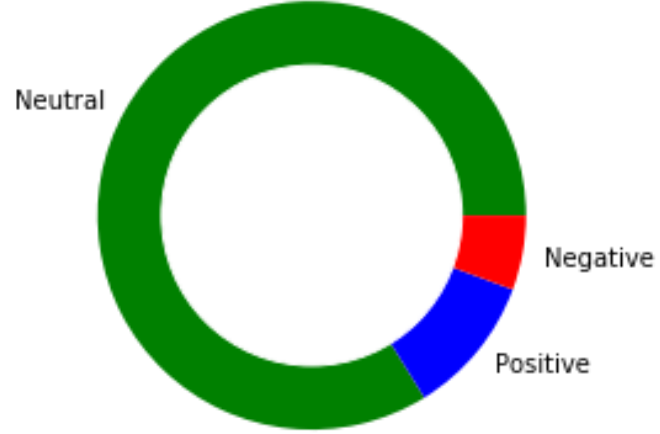

Figure 13 Polarity percentage of bigrams for China Covid-19 vaccination. In this approximately $87.86 \%$ are the neutral statements out of 32,433 corpora, $7.77 \%$ are the positive statements and lastly, $4.37 \%$ are the negative ones.

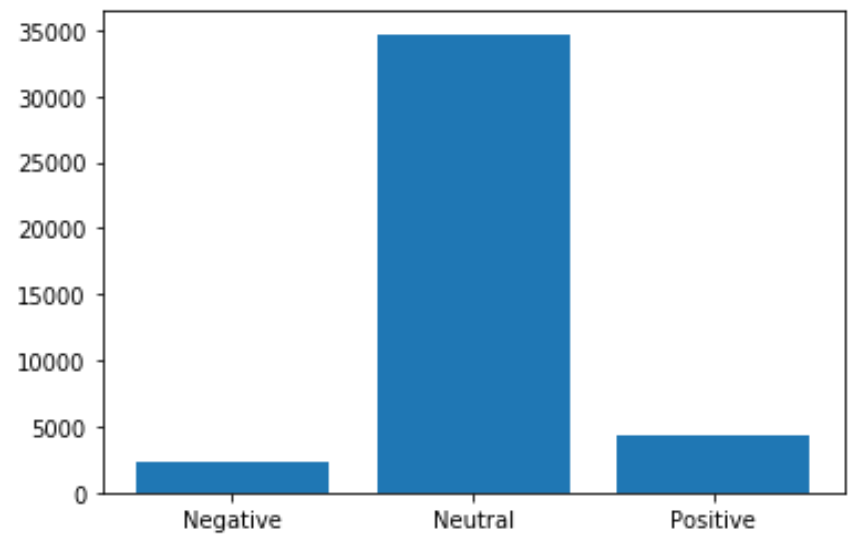

Figure 15 Graphical representation of number of trigrams that has been classified into three different segments negative, positive and neutral in the Chinese Covid-19 vaccination context.

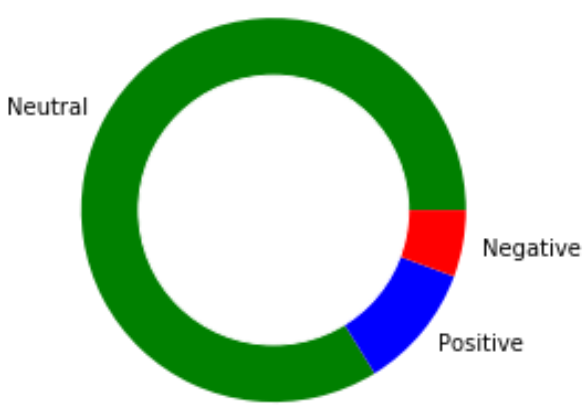

Figure 17 Polarity percentage of trigram for China Covid19 vaccination. In this approximately $83.81 \%$ are the neutral statements out of approximately 35,000 corpora,

$10.57 \%$ are the positive and $5.62 \%$ are the negative statements. 
Last, in the analysis, the frequency of bigrams and trigrams have been observed for both the countries. The top 50 bigrams and trigrams have been plotted with the help of matplotlib library using Python. According to the Indian context (in Fig 18 and Fig 19) vaccines developed by Serum Institute of India is one of the major highlights of the textual data. As per the bar plot, the sentiments are more on the positive sides.

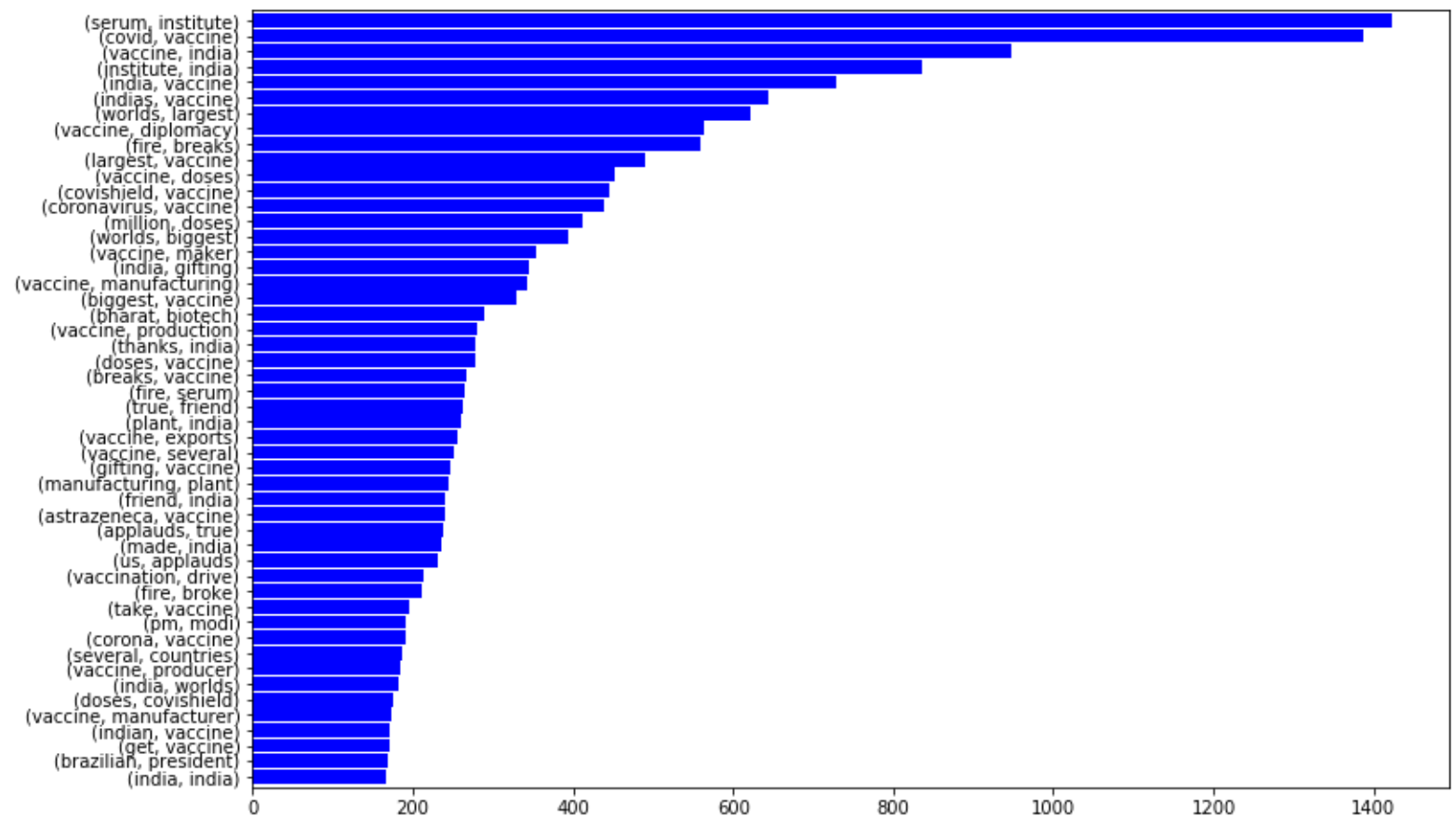

Figure 18 the bigram plot of the 50 most frequently occurring words for India Covid-19 vaccination data. The y axis consists of bigrams and the $x$ axis is the count of words that how many times these pair of words occur together in the corpus.

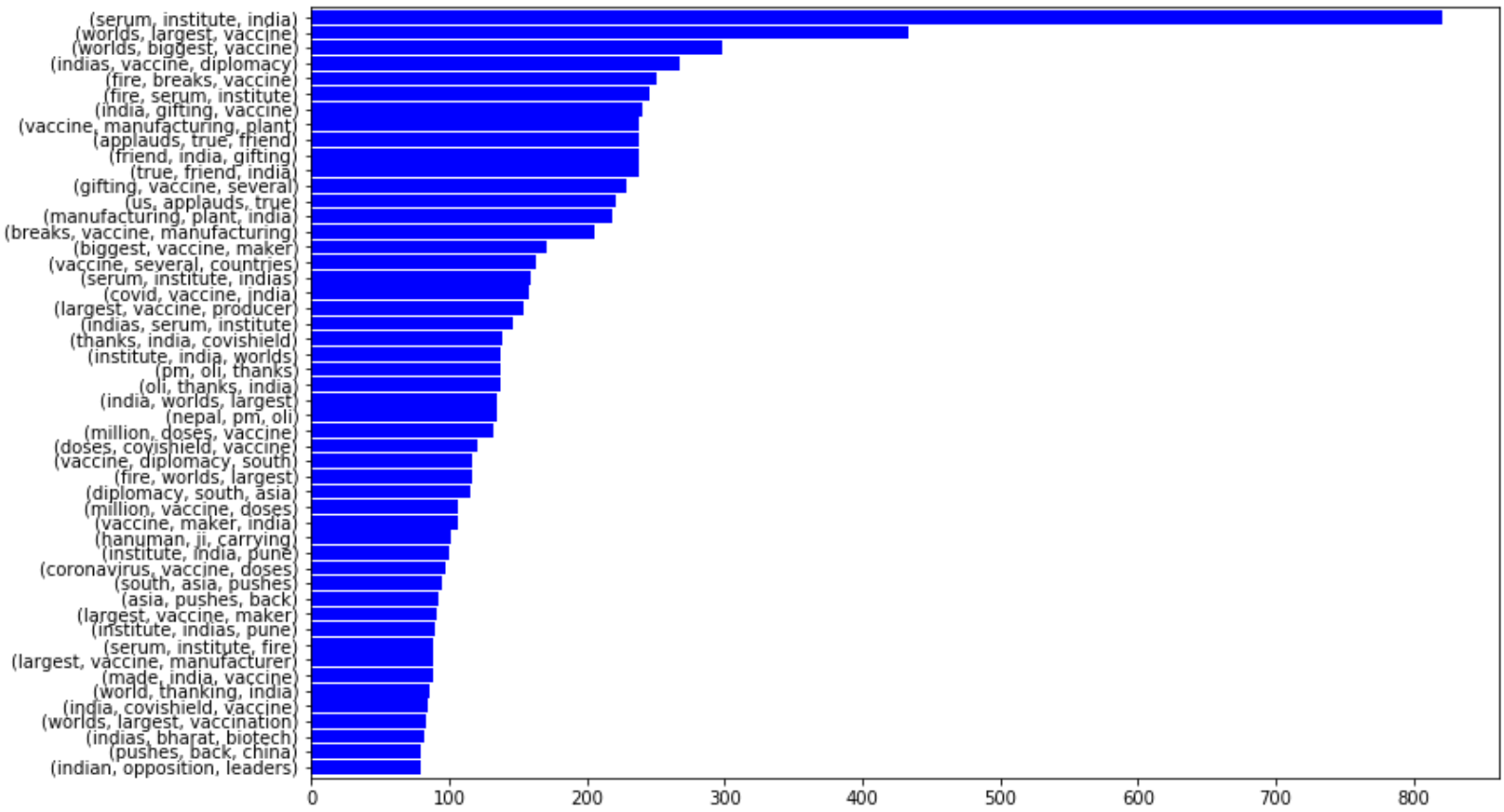

Figure 19 The trigram plot of the 50 most frequently occurring words for India twitter dataset. In this instead of two words, we tokenize the sentence into 3 words or generally called it trigrams which is depicted by $Y$ axis and the $x$ axis depicts the frequency or the count that how many times these trigrams occur or appear together. 
Some of the most commonly occurring words visualized (Fig 20 and Fig 21) in China covid-19 vaccination are sinopharm, sinovac, efficacy rate, India rises vaccine, faulty ppe, world sells faulty, ppe sells unproven, experimental biologics.

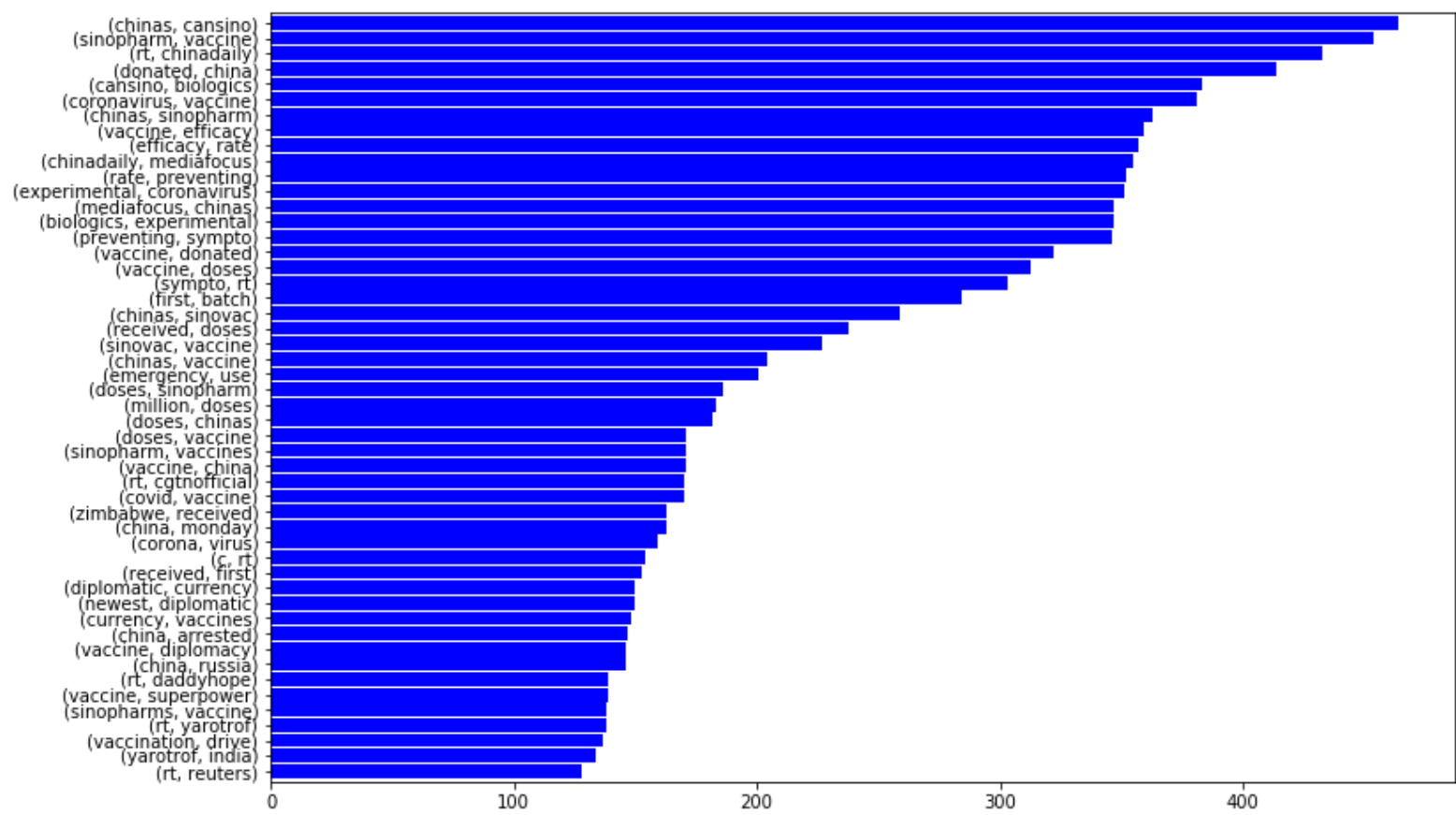

Figure 20 The bigram plot of the 50 most frequently occurring words for China Covid-19 vaccination data. The y axis comprises bigrams and the $x$ axis is the count of words that how many times these pair of words occur together in the corpus.

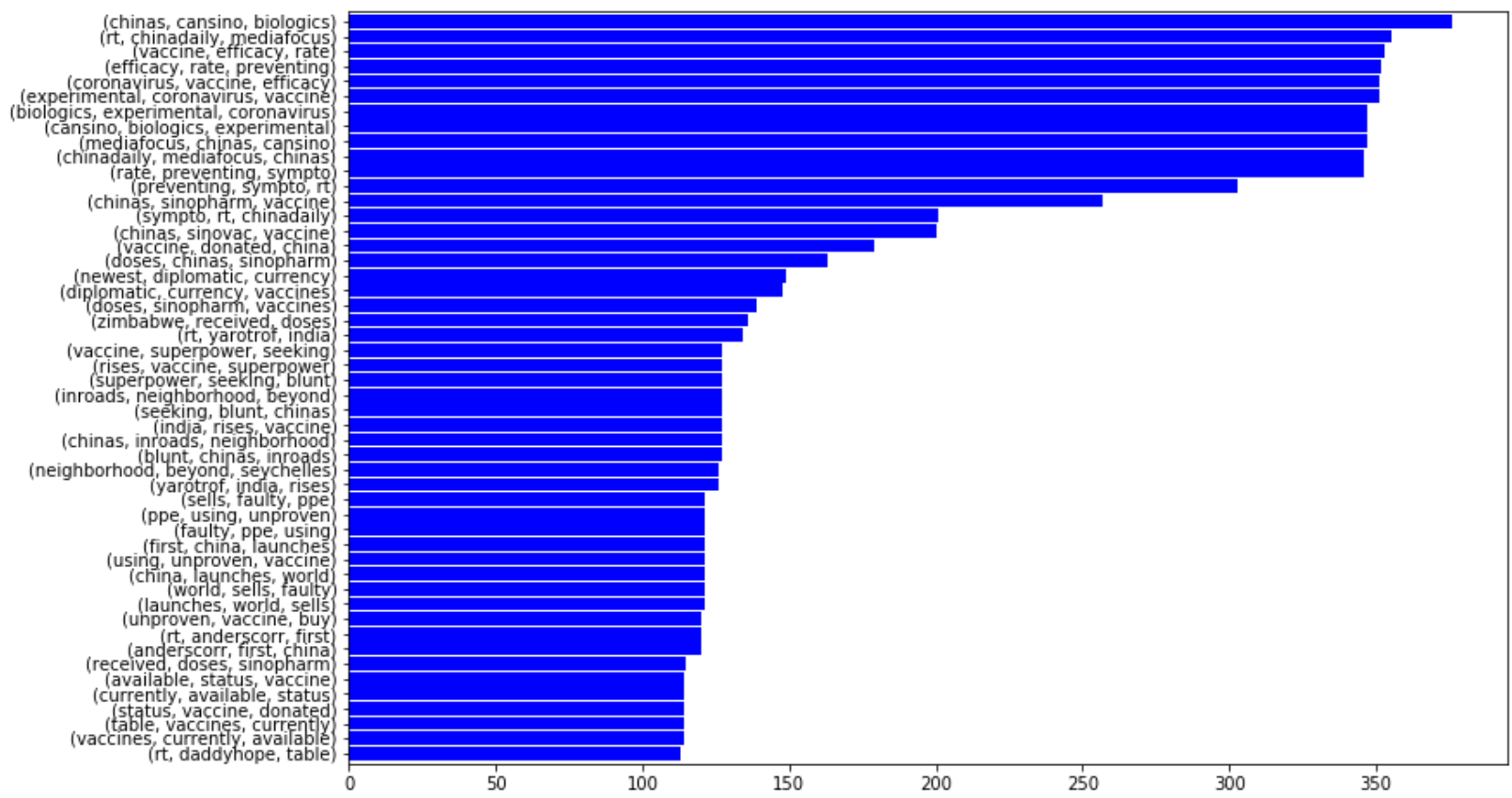

Figure 21 The trigram plot of the 50 most frequently occurring words for China covid-19 vaccination twitter dataset. In this instead of two words, we tokenize the sentence into 3 words also known as trigrams, which is depicted by $Y$ axis and the $x$ axis depicts the frequency or the count that how many times these trigrams occur or appear together. 


\section{Limitations}

The limitations of the study are:

- Because of Twitter API restrictions, we cannot extract the good amount of China covid-19 vaccination data from twitter.

- Only able to analyse the tweets or twitter posts in the English language (mostly the text or emoji). In this research, we are not analysing the images or the videos.

\section{Conclusion}

From the above analysis we can conclude that out of 24,000 approx. tweets, most people have positive sentiments regarding global data for the covid-19 vaccination. $54.948 \%$ of tweets falling under positive segment, $30.25 \%$ having the neutral consensus and rest $14.79 \%$ having negative opinions.

But if we look for the comparative analysis for India and China regarding covid-19 vaccination, India is getting an edge over China in terms of neutral comments, whereas China covid-19 vaccination has more of positive statements (which is approx. 40.42\%) when compare to India covid-19 vaccination twitter data (which is approx. 35.11\%). Besides the positive statements, China is also leading by $1.62 \%$ in negative statements regarding the coronavirus vaccination. If we look for the polarity as per the bigrams and trigrams for both the countries, India is leading in positive and neutral statements by margin from China. Though, most of the positive comments or the tweets for China covid-19 vaccination are coming from Beijing and Pakistan.

\begin{tabular}{|l|c|c|c|}
\hline \multicolumn{4}{|c|}{ Sentiments of People towards Covid-19 vaccination } \\
\hline & $\begin{array}{l}\text { Positive } \\
\text { Sentiment } \\
\text { in \% }\end{array}$ & $\begin{array}{l}\text { Neutral } \\
\text { Sentiment } \\
\text { in \% }\end{array}$ & $\begin{array}{l}\text { Negative } \\
\text { Sentiment } \\
\text { in \% }\end{array}$ \\
\hline Global Dataset & $54.95 \%$ & $30.35 \%$ & $14.79 \%$ \\
\hline India_sentences & $34.11 \%$ & $53.83 \%$ & $12.05 \%$ \\
\hline China_sentences & $40.42 \%$ & $45.90 \%$ & $13.67 \%$ \\
\hline India_bigram & $9.69 \%$ & $86.23 \%$ & $4.08 \%$ \\
\hline China_bigram & $7.77 \%$ & $87.86 \%$ & $4.37 \%$ \\
\hline India_trigram & $9.69 \%$ & $81.46 \%$ & $4.08 \%$ \\
\hline China_trigram & $10.57 \%$ & $83.81 \%$ & $5.62 \%$ \\
\hline
\end{tabular}

